\title{
Erosion Characteristics of Hydraulic Turbine Guide-Vane End Clearance in Sediment Water Flow: A Simplified Model Analysis
}

\author{
Wei Han ${ }^{1,2 *}$, Jie Wang1', Jingbo Kang1, Lianyuan Li $^{3}$, Guoyi Peng ${ }^{4}$ \\ ${ }^{1}$ College of Energy and Power Engineering, Lanzhou University of Tech, Lanzhou, China \\ ${ }^{2}$ Key Laboratory of Fluid Machinery and Systems of Gansu Province, Lanzhou, China \\ ${ }^{3}$ University of Leicester, Leicester, UK \\ ${ }^{4}$ Nihon University, Koriyama, Japan \\ Email: ^hanwei@lut.cn, *peng@mech.ce.nihon-u.ac.jp
}

How to cite this paper: Han, W., Wang, J., Kang, J.B., Li, L.Y. and Peng, G.Y. (2017) Erosion Characteristics of Hydraulic Turbine Guide-Vane End Clearance in Sediment Water Flow: A Simplified Model Analysis. Journal of Flow Control, Measurement \& Visualization, 5, 111-126.

https://doi.org/10.4236/jfcmv.2017.54009

Received: May 9, 2017

Accepted: September 22, 2017

Published: October 24, 2017

Copyright (๑) 2017 by authors and Scientific Research Publishing Inc. This work is licensed under the Creative Commons Attribution International License (CC BY 4.0).

http://creativecommons.org/licenses/by/4.0/

\begin{abstract}
The effect of clearance flow on the erosion characteristics of a circular cylinder with a backward facing step in sediment-laden water flow is analyzed numerically with the mixture model and the re-normalization group (RNG) $k-\mathcal{E}$ turbulence model. Thirty-six monitoring points are set up on different stream surfaces to collect information on the impact erosion under different flow conditions, where the Initial Sediment Volume Fraction (ISVF) is set to 0.05, $0.075,0.1,0.125$, and 0.15 ; particle diameter is set to $0.05 \mathrm{~mm}, 0.15 \mathrm{~mm}, 0.25$ $\mathrm{mm}, 0.35 \mathrm{~mm}$, and $0.45 \mathrm{~mm}$ respectively. The distribution of particle velocity and Local Solid-Phase Volume Fraction (LSVF) along different stream surfaces are calculated, based on which the trend of erosion is qualitatively evaluated. ISVF and particle diameter play different roles on the impact erosion index parameter $\left(c_{s} w_{s}^{3}\right)$ on the different wetted walls. Relative wear rate of numerical estimation agrees well with the practical one under the same working condition. Numerical analysis demonstrates that guide vane with a negative curvature end surface (concave surface) can decrease erosion damage effectively, which may provide a reference for optimal design and maintenance of hydraulic turbine.
\end{abstract}

\section{Keywords}

Erosion Characteristics, Hydraulic Turbine, Guide Vane, Solid-Liquid Two Phase Flow, Numerical Simulation

\section{Introduction}

The guide vane of hydraulic turbine is often subjected to serious abrasion under 
the condition of sediment-laden flow in the Yellow River of China. The clearance flow between the seal surface of the guide vane and the bottom ring (or the head cover) of the hydraulic turbine can be simplified as a flow around circular cylinder (rotating shaft of guide vane) with a backward facing step. Flow separation, reattachment and re-development of sediment-laden water often appear in the two typical flow patterns, which can result in serious erosive damage of the guide vane and shaft as shown in Figure 1. It is widely found in many engineering fields such as hydraulic engineering, chemical engineering and marine engineering, etc.

Erosion in hydraulic machine occurs due to the particle impact and sliding (scouring) action [1] [2]. In dilute solid-liquid flow, erosion is likely to be more due to particle impact [3]. Usually, erosion is predicted via erosion mechanisms including the characteristic of multiphase flow, properties of particle and casing together with certain empirical constants. If properties of the particle and casing material are considered to be known, erosion prediction can be divided into the computation of the multiphase flow field in the relevant component and determination of erosion coefficients. Both impact and sliding erosion coefficients are described elsewhere [1] [4] [5] [6] in the literature also.

Two-dimensional backward facing step are presented to explore the effects of step height and Reynolds number on turbulent separated flow. The effect of the expansion ratio (defined as the ratio of step height to channel height) on the reattachment length is summarized as a function of the Reynolds number. The primary and the secondary recirculation regions expand with the increase of the backward step height and then the maximum turbulent kinetic energy increases [7] [8]. It was found that the large eddy structure also has an important influence on the backward step flow [9].

Four stages of the development of the starting vortex and the process of the pairing and merging of small vortices and the formation of large scale coherent vortices in backward facing step flow have been observed with the particle image velocity (PIV) [10] [11]. The most important finding is that the interaction of the shear layer is an important factor in the formation and shedding of the vortices in the wake region of the cylinder [12] [13]. It was found in the process of starting flow that the effect of impulsive motion is relatively strong and vortex
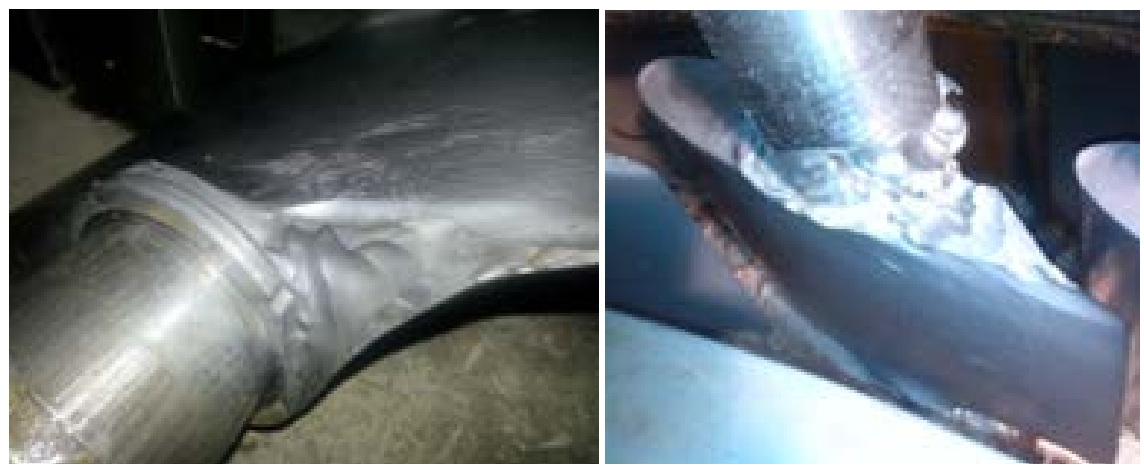

Figure 1. Erosion on turbine guide vanes. 
plays a major role in the whole process [14]. The most serious erosion and the largest probability of sediment incipience occur at the location near the reattachment point [15]. In the gas-particle two-phase flow of the backward facing step, particle motion in the recirculation zone is weaker than that in the fully developed flow region [16]. Numerical simulation have been performed by [17] [18] [19], presented for the detached-eddy simulation (DES) of the flow around a circular cylinder in laminar flow and at a high sub-critical Reynolds number [17] [18]. Large eddy simulation (LES) method and Reynolds average are also applied to simulate drag characteristics at high Reynolds around circular cylinder [19].

In this study, the clearance flow between end surface of guide vane and head cover of the hydraulic turbine is simplified to a flow around circular cylinder with a backward step. The erosive characteristics have been presented with different particle diameter $d$ and ISVF. In particular, ISVF and particle diameter have different effects on the erosion parameter $\left(c_{s} w_{s}^{3}\right)$. Finally, three redesign models with positive, zero and negative curvature are proposed to evaluate the effects of end-surface shape on erosion damage.

\section{Simplified Model for Flow around the Rotating Shaft of a Turbine Guide Vane}

Figure 2 shows the assembly of guide vane related parts of a hydraulic turbine, where an assembly gap between the surface seal of the guide vane and the head cover plate (or bottom ring) is formed. In the operation of hydraulic turbine, sediments flow through the assembly gap with a high speed and cause serious erosion around the guide vane shaft and end surface. In order to investigate the erosion of end clearance, a simplified model for the assembly gap considering the effect on the rotating shaft of the guide vane is given, where the clearance flow is simplified to turbulent multi-phase flow around a circular cylinder with a backward facing step, as shown in Figure 3.

About 36 monitoring points as shown in Figure 3 are located on the step, upper and bottom walls of the downstream step to collect erosion parameters

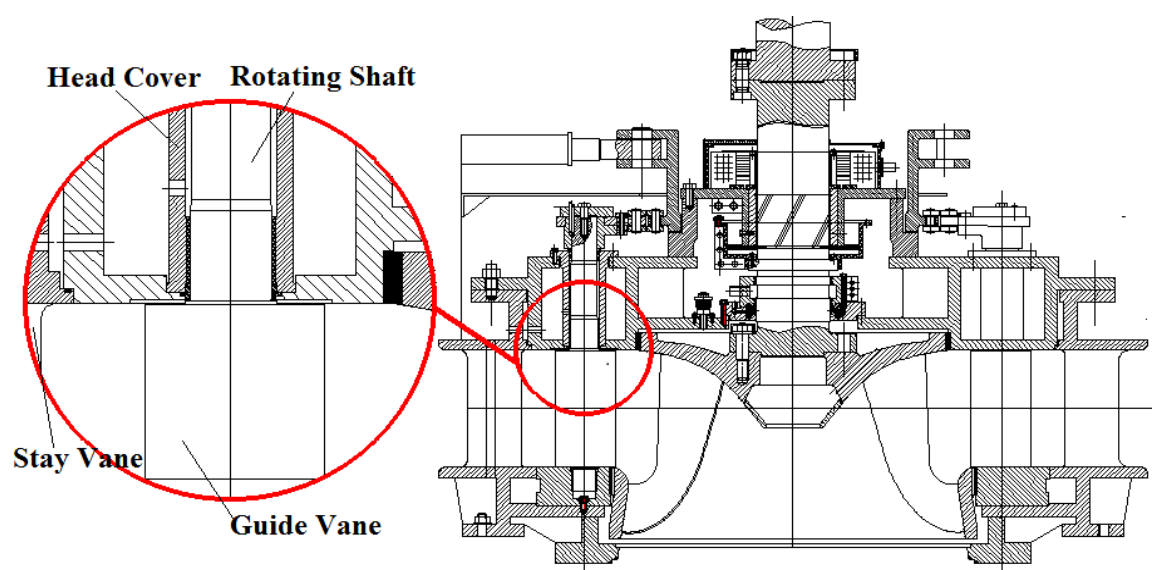

Figure 2. Structure chart of the Francis turbine. 


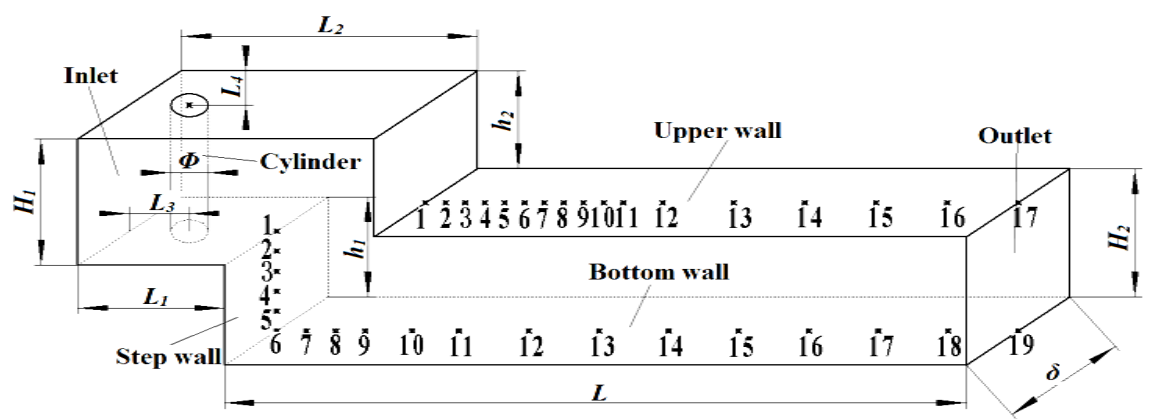

(a)

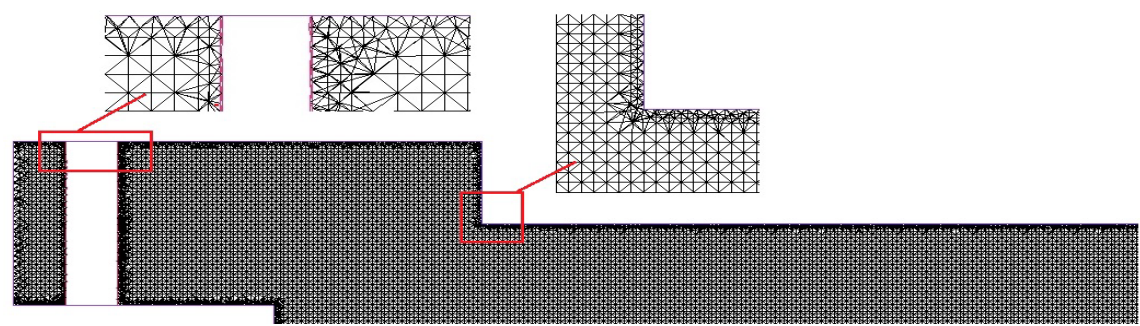

(b)

Figure 3. Geometric model and computational domain. (a) Computational domain; (b) Un-structure computational grid.

information. The geometric parameters of the simplified backward step model are given in Table 1.

\section{Method of Liquid-Solid Two-Phase Flow Simulation}

\subsection{Assumptions}

In consideration of the complexity of clearance flow of sediment-laden water, the following assumptions have been adopted.

1) Particles are spherical with the same diameter $d$.

2) The phase change is negligible.

3) The ISVF $\left(\varphi_{i}\right)$ at the inlet is uniform.

4) All the solid walls are treated as no-slip ones.

\subsection{Model and Equations of Computation}

The effect of turbulent vortices on the flow is considered in the RNG-k- $\varepsilon$ turbulence model, which can provide a good understanding of flow separation and complex secondary flow performance [20]. It has high reliability and economics of computation in engineering turbulence calculation [21]-[26]. The pressure and velocity is coupled by using the semi-implicit method for pressure-linked equations (SIMPLE) algorithm proposed by Patankar [27].

The mixture model is a simplified Euler model used to simulate sediment-laden water flow. It can be used for numerical calculation of dilute solid-liquid flow as the particle Stokes number $S t<<1$ coinciding with the actual working condition. So, the Mixture model and RNG $k-\mathcal{E}$ turbulence model are used to simulate the complex flow field of the sediment-laden water in the clearance. 
Table 1. The geometric parameters of the backward-step flow around a cylinder.

\begin{tabular}{cc}
\hline$H_{1}=40 \mathrm{~mm}$ & $L_{2}=90 \mathrm{~mm}$ \\
$L_{1}=50 \mathrm{~mm}$ & $L_{4}=15 \mathrm{~mm}$ \\
$L=300 \mathrm{~mm}$ & $L_{3}=15 \mathrm{~mm}$ \\
$\delta=30 \mathrm{~mm}$ & $\Phi=10 \mathrm{~mm}$ \\
$H_{2}=40 \mathrm{~mm}$ & $h_{1}=h_{2}=20 \mathrm{~mm}$ \\
\hline
\end{tabular}

The continuity equation and the momentum equation of the mixture model are given as

$$
\begin{gathered}
\frac{\partial \rho_{f}}{\partial t}+\nabla \cdot\left(\rho_{f} u_{f}\right)=0 \quad \text { (No phase transformation) } \\
\rho_{f}\left[\frac{\partial u_{f}}{\partial t}+\left(u_{f} \cdot \nabla\right) u_{f}\right]=\rho_{f} g-F+\nabla \cdot\left[\left(1-\alpha_{s}\right)\right] T_{f} \\
\frac{\partial \rho_{s}}{\partial t}+\nabla \cdot\left(\rho_{s} u_{s}\right)=0 \text { (No phase transformation) } \\
\rho_{s}\left[\frac{\partial u_{s}}{\partial t}+\left(u_{s} \cdot \nabla\right) u_{s}\right]=\rho_{s} g-F+\nabla \cdot\left(\alpha_{s} T_{s}\right)
\end{gathered}
$$

where subscripts $f$ and $s$ indicate the fluid and the solid phase, $\alpha$ does the volume fraction, $\rho$ the density, $u$ the velocity, $g$ the gravitational acceleration, $F$ the two-phase interaction force per unit volume, and $T$ the stress tensor, respectively.

\subsection{Initial and Boundary Conditions}

Initial and boundary conditions are set as follows:

1) Solid-phase particle density $\rho_{s}=2580 \mathrm{~kg} / \mathrm{m}^{3}$.

2) The velocity at the inlet boundary: $V_{l}=3 \mathrm{~m} / \mathrm{s}$.

3) The ISVF $\left(\varphi_{i}\right)$ is defined to be $0.05,0.075,0.1,0.125$, and 0.15 at the inlet, respectively.

4) The particle diameter $d$ is given to be $0.05 \mathrm{~mm}, 0.15 \mathrm{~mm}, 0.25 \mathrm{~mm}, 0.35$ $\mathrm{mm}$ and $0.45 \mathrm{~mm}$, respectively.

5) The free flow condition is applied to the outlet.

6) The standard wall function is applied to all the solid walls.

\section{Calculation Result and Discussion}

In order to clarify the mesh independence of numerical results, three meshes of different number density were used and the dependence of computation results is investigated. Figure 4 shows, as a sample, the distribution of LSVF $\left(\varphi_{1}\right)$ obtained with different cell numbers along the six monitoring position located at the step wall, which is closed to a flow separation region and numerical result is easily affected by the solution of computation mesh.

As shown in Figure 4, the $\varphi_{1}$ distributions converge with the increasing of cell numbers. When the cell number increases from $12.3 \times 10^{5}$ to $18.8 \times 10^{5}$, the 


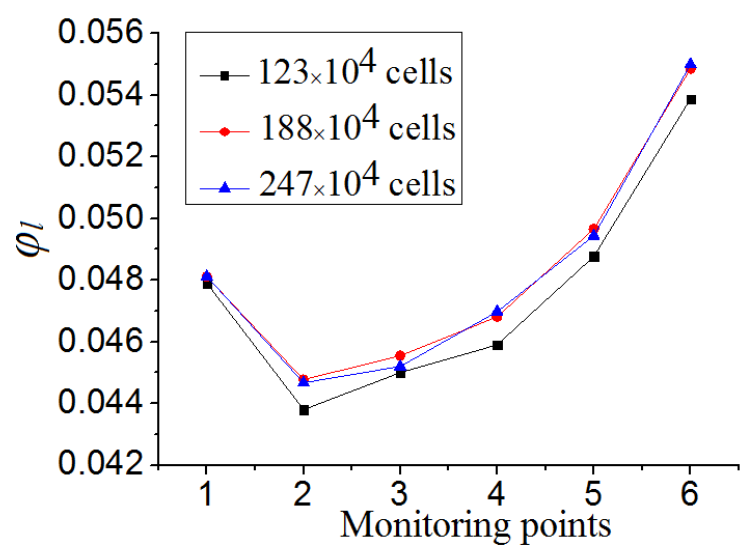

Figure 4. Confimation of mesh independence: LSVF distributions at the step wall.

maximum variation of $\varphi_{1}$ is about $2.5 \%$. However, when the cell number is increased from $18.8 \times 10^{5}$ to $24.7 \times 10^{5}$ the maximum variation of $\varphi_{1}$ is only about $0.5 \%$. Thus the solution of mesh with $18.8 \times 10^{5}$ cells is thought to be enough and has been adopted in the flowing simulations.

\subsection{Solid-Phase Flow Structure}

Figure 5 shows the solid-phase streamlines under different initial conditions, where the color distinguishes the value of velocity as shown in the label.

Flow separation, reattachment, and redevelopment occur behind the two steps. Recirculation zones I and II, as well as reattachment zones I and II appear at the bottom and the upper walls of the downstream step. Behind the two steps, two vortices with different sizes are induced at the recirculation zones I and II. The size of the vortex near the upper wall is larger than the vortex on the bottom wall along the flow direction. In other words, the reattachment point on the upper wall lies posterior to the bottom wall along the flow direction. The flow develops fully in the downstream and flow lines become relatively smooth behind the two recirculation zones step by step.

\subsection{Distributions of Solid-Phase Velocity and Volume Fraction}

The impact erosion rate parameters include the particle velocity $w_{s}$ and LSVF along the wall. In consideration of the impact erosion rate the variations of $w_{s}$ and LSVF are investigated. Firstly, the clearance flow has been simulated with different ISVF $(0.05,0.075,0.10,0.125$ and 0.15$)$ when the particle diameter $d$ is $0.05 \mathrm{~mm}$. Figure 6 and Figure 7 respectively show the variations of representative particle velocity $w_{s}$ and LSVF along the surface walls.

\subsubsection{Distributions of Particle Velocity}

As shown in Figure 6(a), the tendency of velocity variation on the upper wall matches "W-shaped" closely. The effect of the vortex in recirculation zone II consumes the energy of the particles, resulting in a gradual decrease in the velocity. Behind the reattachment zone, the particle-carrying capacity of the fluid 

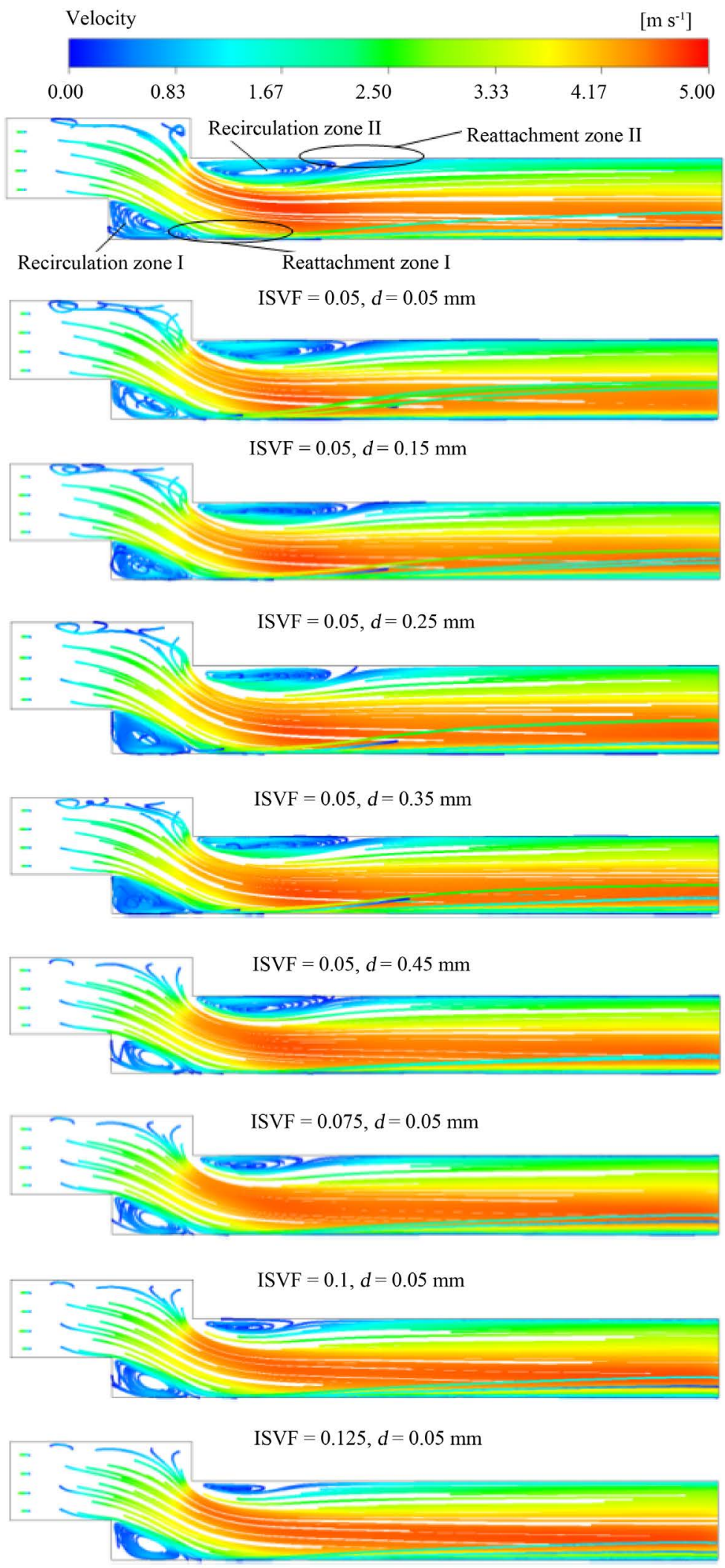

ISVF $=0.15, d=0.05 \mathrm{~mm}$

Figure 5. The flow field around a circular cylinder with a backward facing step. 


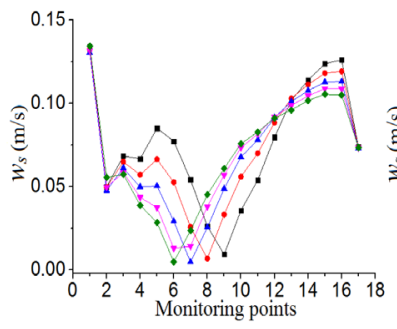

(a)

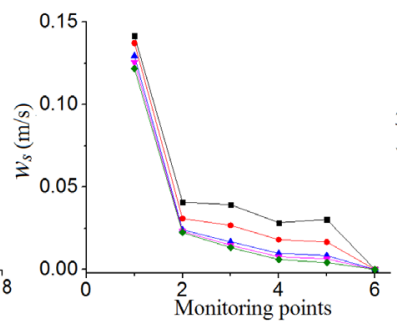

(b)

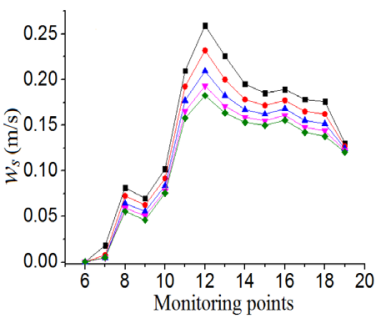

(c)

Figure 6. Velocity distribution of solid-phase with different ISVF. (a) Upper wall; (b) Step wall; (c) Bottom wall.

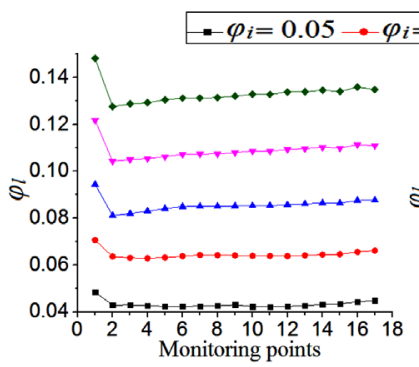

(a)

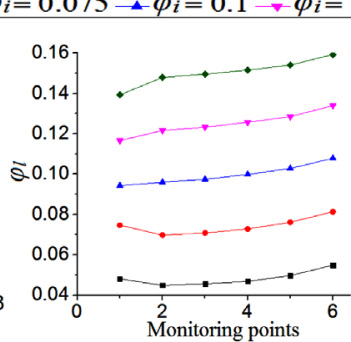

(b)

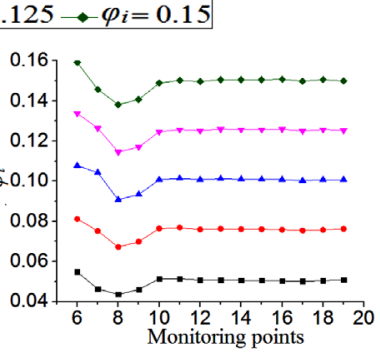

(c)

Figure 7. LSVF distributions under different ISVF. (a) Upper wall; (b) Step wall; (c) Bottom wall.

is enhanced by the upwelling, which causes the velocity of particles to increase first and then decrease. The position of particle minimum speed gradually moves from the reattachment zone and to the point 1 with the increasing of ISVF. The velocity of particle increases initially and then decreases, and reaches the maximum at point 16 behind the recirculation zone II.

As shown in Figure 6(b), the vortex in the recirculation zone I plays an important role on the aggregation of solid particles with higher speed at the edge of the vortex, which forms the disc-cutting effect on the step wall. The velocity of particles on the step wall gradually decreases from top to bottom which leads to particle accumulation at the bottom of the step. The velocity of particles at point 1 is higher than other points $2-6$ as the monitoring point 1 is affected by the mainstream rather than the vortex.

As shown in Figure 6(c), the velocity of particles on the bottom wall increases first and then decreases. It reaches the maximum at the reattachment point. With the increasing of ISVF, particle velocity decreases at the same monitoring point.

\subsubsection{Distributions of Solid Phase Volume Fraction}

Figure 7 shows the distribution of LSVF at different monitoring points. The LSVF decreases firstly and then slowly increases along the direction of mainstream on the upper wall as shown in Figure 7(a). The tendency of variation on the bottom wall closely matches to that on the upper wall as shown in Figure 7(c). 
In Figure 7(b), the LSVF increases linearly from the top to the bottom of the step with increasing of ISVF.

\subsection{Effect of Particle Diameter}

The velocity distribution and the LSVF of particles are investigated under different particle diameters $(0.05 \mathrm{~mm}, 0.15 \mathrm{~mm}, 0.25 \mathrm{~mm}, 0.35 \mathrm{~mm}$, and $0.45 \mathrm{~mm})$ when the ISVF is set to 0.05 and the results are shown in Figure 8 and Figure 9 , respectively.

As shown in Figure 8 and Figure 9, with the increase of particle diameter the particle Stokes number increases and particle's following behavior reduces. Particle inertia, vortex and upwelling play a coupling role on the LSVF and the particle velocity distribution. Behind the reattachment zone I, particles in the mainstream surfaces gradually move to the bottom of the step with increasing of LSVF at the same monitoring point, which is contrary to the situation on the upper wall.

\subsection{Estimation of Particle Impact Erosion}

Erosion of the wetted component can be attributed to the particle impact and abrasive erosion, described by Equation (5) [28].

$$
W_{s p}=\theta(\alpha) \rho c_{s} w_{s}^{m}
$$

where the exponent $m$ is given to be $3, \rho$ (assumed constant) denotes the density of the solid particles in $\mathrm{kg} / \mathrm{m}^{3}, c_{s}$ the LSVF, and $w_{s}$ the particle relative velocity in

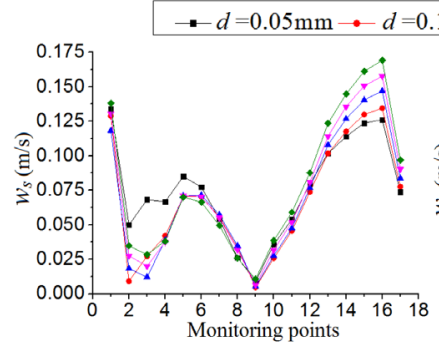

(a)

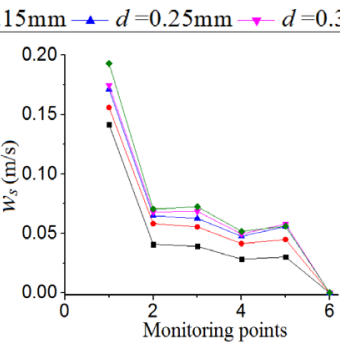

(b)

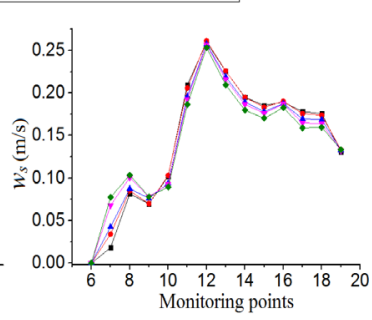

Figure 8. Solid-phase velocity distribution with different solid particle diameters. (a) Upper wall; (b) Step wall; (c) Bottom wall.

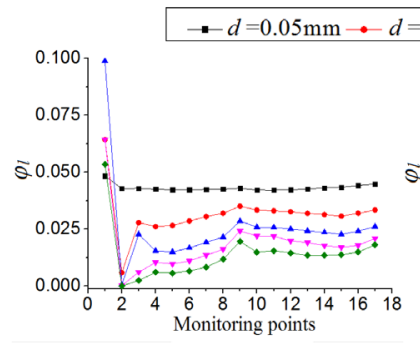

(a)

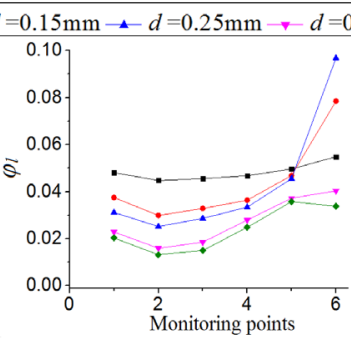

(b)

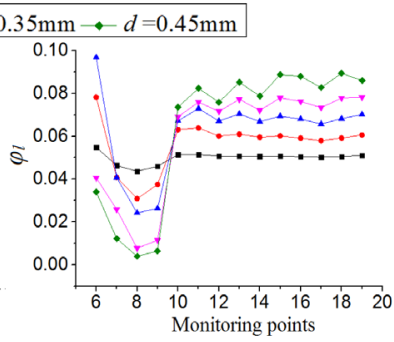

(c)

Figure 9. LSVF distribution with different solid particle diameters. (a) Upper wall; (b) Step wall; (c) Bottom wall. 
$\mathrm{m} / \mathrm{s}$. The expression $\theta(\alpha)$ accounts for the effects of particle diameter, the material of the over current component and variations for different combinations and angles.

The abrasive erosion with the slide action is very weak and erosion is likely to be more due to particle impact in dilute solid-liquid flow [28]. The abrasive erosion of the particles cannot be considered for the wetted components. So, the specific erosion rate has been described for a particle at speed $w_{s}$ hitting the wall with an angle of $\alpha$ [29]. As the impact angle $\alpha$ varies marginally at the reattachment points of the upper wall, step wall and bottom wall, the variations in the parameter $\theta(\alpha)$ are bound to be marginal too [28]. Therefore, it would be sufficient to compare the value of index parameter $C_{s} w_{s}^{3}$ along the monitoring wall in the study for the prediction of impact erosion rate.

The impact wear index parameter $\left(c_{s} w_{s}^{3}\right)$ including the velocity of particles and LSVF are investigated along the monitoring wall with different ISVF and particle diameters, as shown in Figure 10 and Figure 11.

\subsubsection{Impact Wear Index Parameter under Different ISVF $\left(\varphi_{i}\right)$}

From Figure 10, it is found that the larger the ISVF is, the higher the value of $c_{s} w_{s}^{3}$ is on the whole wetted wall. The curves of impact wear index $\left(c_{s} w_{s}^{3}\right)$ approximately present into be inverted S-shape, L-shape and inverse $\mathrm{V}$-shape along the upper wall, step wall and bottom wall, respectively.

On the upper wall, the most serious erosion occurs at the point 1. From point 7 to point 18 , the impact wear index increases gradually and then decreases. It

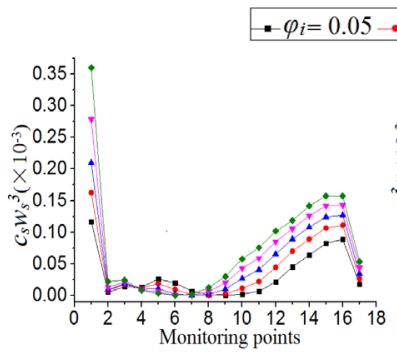

(a)

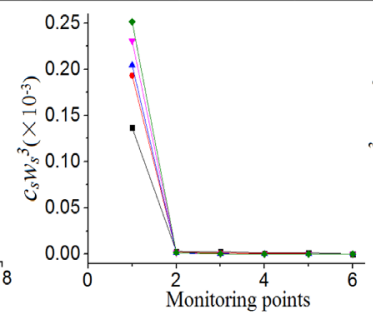

(b)

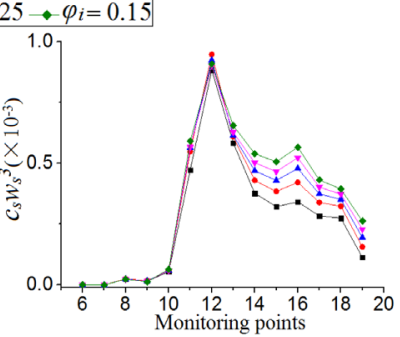

(c)

Figure 10. Comparison of erosion trends for different ISFV. (a) Upper wall; (b) Step wall; (c) Bottom wall.

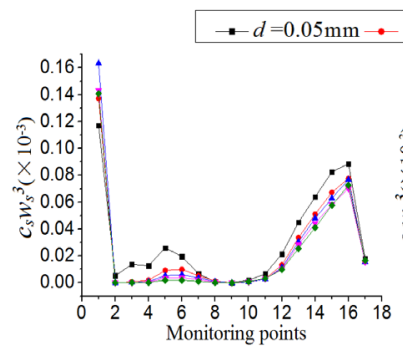

(a)

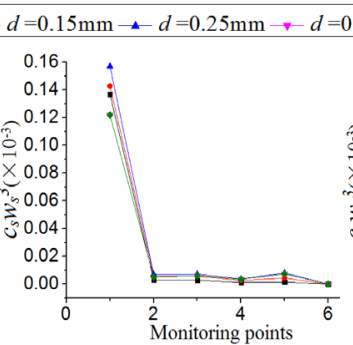

(b)

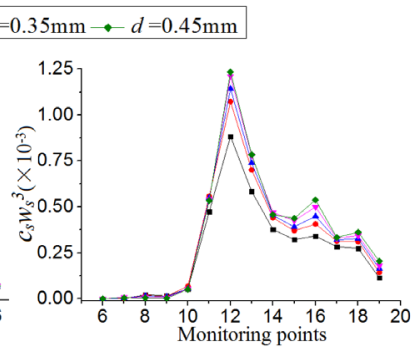

(c)

Figure 11. Comparison of erosion trends for different particle diameters. (a) Upper wall; (b) Step wall; (c) Bottom wall. 
has the maximum peak value near the point 16.On the bottom wall, the most serious erosion occurs at the reattachment point (near the point 12). From point 8 to point 20 , the impact wear index increases gradually and then decreases. On the step wall, the impact wear index decreases gradually and the most serious erosion occurs at the point 1 .

\subsubsection{Impact Wear Index with Different Particle Diameters}

As shown in Figure 11, the curves of impact wear index $\left(c_{s} w_{s}^{3}\right)$ under different particle diameters present to be approximately an inverted S-shape, an L-sharp and an inverse $\mathrm{V}$-shape along the upper wall, the step wall and the bottom wall, respectively. But the effects of particle diameter on the $c_{s} w_{s}^{3}$ are different on the different wetted walls.

On the upper wall the impact wear index increases gradually and decreases from the point 2 to the point 8 and shows the maximum peak values near the point 5 for the effect of vortex induced by the recirculation II. At the same monitoring point on the upper wall, $c_{s} w_{s}^{3}$ decreases gradually with the increasing of particle diameter between the point 2 and point 18, shown as Figure 11(a). On the contrary, the impact wear index increases gradually with the increasing of particle diameter along the flow direction of bottom wall behind the reattachment point 12, shown as Figure 11(c). Near the point 1 of the upper wall, the effect of particle diameter on the value of $c_{s} w_{s}^{3}$ is nonlinear. It reaches the maximum peak value with the particle diameter $d=0.25 \mathrm{~mm}$, which is the same as the starting point 1 of the step, shown as Figures 11(a)-(c).

\subsubsection{Comparison of Numerical Simulation Results with Actual Erosion}

Figure 12(a) shows the image of end surface erosion of a guide vane (point 6-19) after 6800 hours' operation when the average solid volume fraction $\varphi_{i}=$ 0.05 and diameter $d=0.05 \mathrm{~mm}$. The upper seal of guide vane corresponds to the bottom wall of simplified model. For comparison of practical erosion and calculated result the calculated relative wear rate $(S R W R)$ and actuality relative wear rate $(A R W R)$ are respectively defined as:

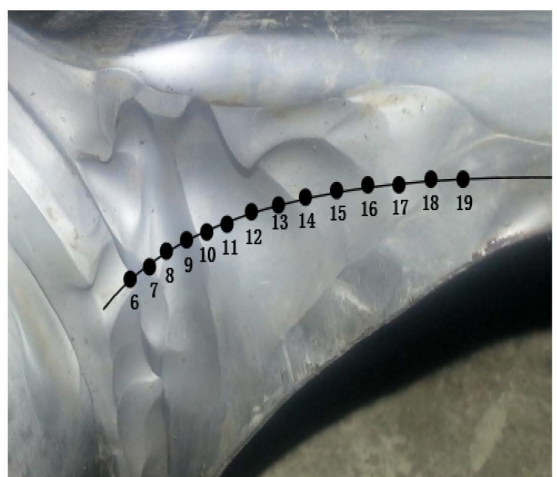

(a)

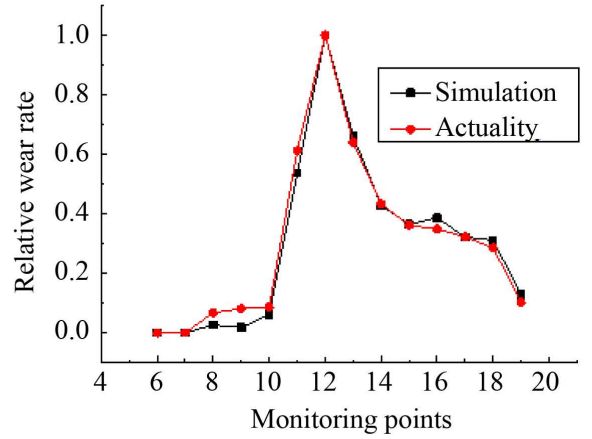

(b)

Figure 12. Erosion on the guide-vane end surface. (a) Image of practical erosion, (b) Comparison of relative erosion rate. 


$$
\begin{gathered}
S R W R=\frac{\left(c_{s} w_{s}^{3}\right)_{i}}{\max \left[\left(c_{s} w_{s}^{3}\right)_{i}\right]},(i=6-19) \\
A R W R=\frac{h_{i}}{\max \left(h_{i}\right)},(i=6-19)
\end{gathered}
$$

where the subscript $i$ indicate an monitoring point, $\left(c_{s} w_{s}^{3}\right)_{i}$ does the impact wear index $\left(c_{s} w_{s}^{3}\right)$ of the monitoring point when ISVF and particle diameter $d$ are set to 0.05 and $0.05 \mathrm{~mm}$, respectively. $h_{i}$ denotes the erosion depth at monitoring point $i$ on the upper surface of guide vane after the practical engineering operation. Figure 12(b) shows a comparison of the relative wear rates of calculated and practical results.

The tendency of relative wear rate and the maximum wear position of guide vane experienced the practical operation, are similar to the results of numerical simulation. The maximum wear occurs at the monitoring point 12 . The calculation results agree to the practice experiment values within an acceptable error range $(-14.9 \%-10.6 \%)$ except at the points 7,8 and 9 on the end surface (bottom wall). The increase of erosion damage in the recirculation zone may be due to the underestimate of disc-cutting effect generated by the step vortex.

\section{Redesign}

According to the above numerical simulation results we know that the maximum impact wear rate appears near the reattachment point closed to the vortex edge area in the recirculation zone. In other word, it should be possible to decrease the impact wear index by decreasing the impact velocity and eliminating the step vortex. So three redesign models with positive, zero and negative curvatures are proposed and they as respectively shown in Figure 13.

Figure 14 shows a comparison of erosion index of the redesign models and the initial model under the practical working condition $\left(\varphi_{i}=0.05, d=0.05 \mathrm{~mm}\right)$. It can be found that the erosion damage along the end surface with negative curvature becomes much lesser and uniform compared to any other models. The average impact wear index is $0.18 \times 10^{-3}$, which is decreased by $32.76 \%$ compared to the original model. According to the variations of wear rate, we understand that the end surface of guide vane with negative curvature matches to the streamlines and it can convert impact wear into scouring wear and then avoid the disc-cutting effect possibly. In other words, the guide vane with end surface of negative curvature may decreases the erosion damage and increases its operation life, which was verified by the guide vane with concave end surface [30].

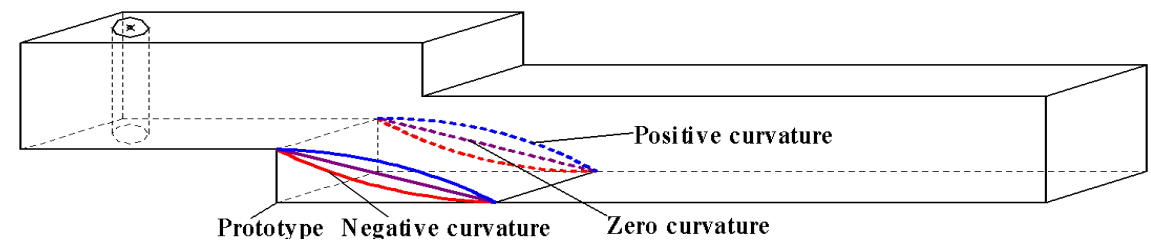

Figure 13. Schematic diagram of three redesign models. 


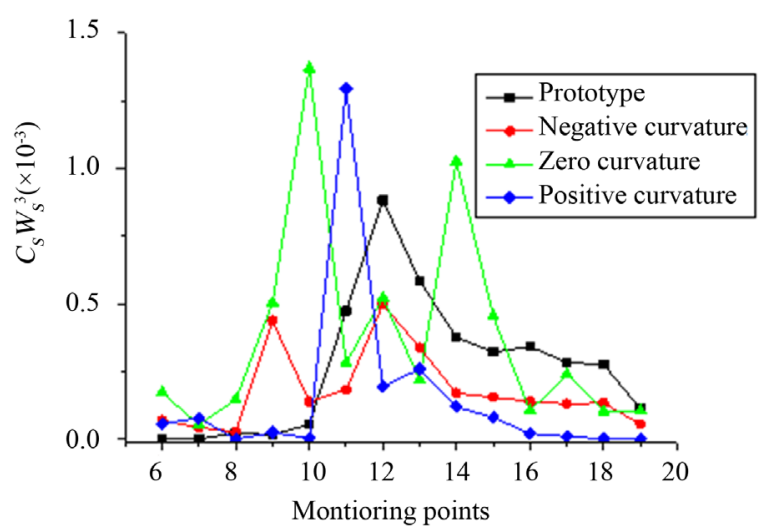

Figure 14. Comparison of erosion tendencies on the bottom wall of different models.

\section{Conclusions}

The flow structure, the particle velocity distribution and LSVF in guide vane end clearance of hydraulic turbine have been investigated in this paper. The impact wear index $\left(c_{s} w_{s}^{3}\right)$ of a circular cylinder with backward facing step in sediment-laden water flow is predicted under different ISVF and particle diameters. Impact wear indexes of three redesign models of the guide-vane end surface are further evaluated under the practical working condition. Summarizations are given as follows:

1) The tendency of impact wear index $\left(c_{s} w_{s}^{3}\right)$ curve presents to be approximately inverted S-shape, L-shape and inverse V-shape along the upper wall, the step wall and the bottom wall, respectively.

2) With the increasing of ISVF, the impact wear index $\left(c_{s} w_{s}^{3}\right)$ increases gradually on the whole wetted wall except in the recirculation zone I and II. The impact wear index decreases gradually with the increasing of particle diameter behind the starting point of the upper wall. On the contrary, $c_{s} w_{s}^{3}$ increases gradually with the increasing of particle diameter behind the reattachment point of bottom wall, which is similar to the step wall.

3) ISVF and particle diameter have different effects on the impact wear index $\left(c_{s} w_{s}^{3}\right)$. On the upper wall and the bottom wall near to the recirculation zone, ISVF plays a major role on the erosion damage. The particle diameter is likely to be a more significant factor for the erosion damage on the step and the bottom walls behind the recirculation zone.

4) Negative curvature surface (concave surface) for the end wall surface of guide vane may decrease the erosion damage effectively.

5) The simplified model has been demonstrated to be effective for predicting the erosion of guide vane system.

\section{Acknowledgements}

This work has been supported by the National Natural Science Foundation of China (Grant No. 51579125 and Grant No. 51669012). The authors would like to 
extend their gratitude to Jiuquan Sanyuan Hydropower Development Company LTD., China for their support to experimental test.

\section{References}

[1] Roco, M.C., Nair, P., Addie, G.R., et al. (1984) Erosion of Concentrated Slurry in Turbulent Flow. ASME FED, 13, 69-77.

https://www.researchgate.net/publication/292004812_EROSION_OF_CONCENTR ATED_SLURRIES_IN_TURBULENT_FLOW

[2] Addie, G.R. and Pagalthivarthi, K.V. (1989) Prediction of Dredge Pump Shell Erosion//Proc. WODCON XII, 12 th World Dredging Conference, World Organization of Dredging Associations, Arlington, 481-504.

https://www.researchgate.net/publication/292706063_Prediction_of_dredge_pump _shell_erosion

[3] Minemura, K. and Uchiyama, T. (1990) Calculation of the Three-Dimensional Behaviour of Spherical Solid Particles Entrained in a Radial-Flow Impeller Pump. Proceedings of the Institution of Mechanical Engineers, Part C: Mechanical Engineering Science, 204, 159-168.

http://journals.sagepub.com/doi/abs/10.1243/pime_proc_1990_204_092_02 https://doi.org/10.1243/PIME_PROC_1990_204_092_02

[4] Pagalthivarthi, K.V. and Helmly, F.W. (1992) Applications of Materials Erosion Testing to Solids Transport via Centrifugal Slurry Pumps//Erosion Testing of Advanced Materials. ASTM International. https://www.astm.org/DIGITAL_LIBRARY/STP/PAGES/STP23861S.htm

[5] Tian, H.H., Addie, G.R. and Pagalthivarthi, K.V. (2005) Determination of Erosion Coefficients for Erosive Erosion Prediction through Coriolis Erosion Testing. Erosion, 259, 160-170. http://www.sciencedirect.com/science/article/pii/S0043164805002012

[6] Clark, H.M.I., Tuzson, J. and Wong, K.K. (2000) Measurements of Specific Energies for Erosive Erosion Using a Coriolis Erosion Tester. Erosion, 241, 1-9. http://www.sciencedirect.com/science/article/pii/S0043164800003276 https://doi.org/10.1016/S0043-1648(00)00327-6

[7] Thangam, S. and Knight, D.D. (1989) Effect of Step Height on the Separated Flow Past a Backward Facing Step. Physics of Fluids A Fluid Dynamics, 1, 604-606. https://doi.org/10.1063/1.857430

[8] Chen, Y.T., Nie, J.H., Armaly, B.F. and Hsieh, H.T. (2006) Turbulent Separated Convection Flow Adjacent to Backward-Facing Step-Effects of Step Height. International Journal of Heat \& Mass Transfer, 49, 3670-3680.

http://www.sciencedirect.com/science/article/pii/S0017931006001633

https://doi.org/10.1016/j.ijheatmasstransfer.2006.02.024

[9] Lian Q. (1993) Experimental Study on the Turbulent Coherent Structure of Backward Step. Acta Mechanica Sinica, 25, 129-133. http://lxxb.cstam.org.cn/CN/abstract/abstract140382.shtml

[10] Ma, Y. and Shen, G. (1996) Investigation on the Properties and Structures of Starting Vortex Flow around 2-d Backward-Step by PIV. Acta Aeronautica et Astronautica Sinica, 17, 257-264. http://hkxb.buaa.edu.cn/CN/abstract/abstract11554.shtml

[11] Liu, Y., Piao, Y. and Cheng, X. (2005) Experimental Investigation on the Turbulent Separated and Reattaching Flow over Backward-Facing Step. Journal of Shanghai Jiao Tong University, 39, 810-812. http://www.cqvip.com/qk/92944x/200505/15985860.html 
[12] Qi, E., Huang, M., Li, W., Li, G., Zhang, X. and Wu, J. (2004) Investigation of the Starting Flow over a Backward Facing Step Flow via PIV. Journal of Hydrodynamics, 19, 533-539. http://www.cqvip.com/qk/94099x/20044/10255046.html

[13] Zhang, X. and Zhou, L. (2004) Pdpa Measurements of the Effect of Wall Roughness on Gas-Particle Flows in a Horizontal Back Ward-Facing Step. Journal of Engineering Thermophysics, 25, 71-74. http://jetp.iet.cn/CN/abstract/abstract4406.shtml

[14] Deng, L. (2009) The Sediment's Incipience Mechanism under Complex Flow. Hunan University. http://cdmd.cnki.com.cn/Article/CDMD-10532-2009164750.htm

[15] Zhang, X., Jiang, J., Jia, Y., Ma, H. and Xiao, Y. (2005) Measurements of Cylinder's Wake by PIV. Journal of Experiments in Fluid Mechanics, 19, 74-78. http://www.syltlx.com/CN/abstract/abstract9391.shtml

[16] Soria, J. (1996) An Investigation of the near Wake of a Circular Cylinder using a Video-Based Digital Cross-Correlation Particle Image Velocimetry Technique. EXperimental Thermal \& Fluid Science, 12, 221-233. http://www.sciencedirect.com/science/article/pii/0894177795000860

[17] Mockett, C., Perrin, R. and Reimann, T. (2010) Analysis of Detached-Eddy Simulation for the Flow around a Circular Cylinder with Reference to PIV Data. Flow, Turbulence and Combustion, 85, 167-180.

https://link.springer.com/chapter/10.1007/978-1-4020-9898-7_36 https://doi.org/10.1007/s10494-009-9243-x

[18] Chang, S., Wang, Y. and Pang, Z. (2009) Numerical Simulation of Flow around Circular Cylinder using SST DES Model. Ship Science and Technology, 23, 30-33. http://jckx.cbpt.cnki.net/WKE/WebPublication/paperDigest.aspx?paperID=616E34 3B-9007-429D-A07E-D620CDB65683

[19] Zhao, M., Mao, J. and Xi, Y. (2015) Research on Drag Characteristic of Flow around Finite Circular Cylinder at High Reynolds Numbers. Journal of Mechanical Engineering, 51, 176-182. https://doi.org/10.3901/JME.2015.22.176 http://www.cjmenet.com.cn/Jwk_jxgcxb/CN/abstract/abstract10512.shtml

[20] Yakhot, V. and Orszag, S.A. (1986) Renormalization Group Analysis of Turbulence. I. Basic Theory. Journal of Scientific Computing, 1, 3-51. https://journals.aps.org/prl/abstract/10.1103/PhysRevLett.57.1722 https://doi.org/10.1007/BF01061452

[21] Speziale, C.G. (1987) On Nonlinear k-l and k-Models of Turbulence. Journal of Fluid Mechanics, 178, 459-475.

https://www.cambridge.org/core/journals/journal-of-fluid-mechanics/article/on-no nlinear-kl-and-k-models-of-turbulence/7BF348DEA4E7062CA7BD46D021E05F77 https://doi.org/10.1017/S0022112087001319

[22] Mompean, G. (1998) Numerical Simulation of a Turbulent Flow near a Right-Angled Corner using the Specialized Non-Linear Model with RNG k- $\varepsilon$, Equations. Computers \& Fluids, 27, 847-859. http://www.sciencedirect.com/science/article/pii/S0045793098000048

[23] Tutar, M. and Holdø, A.E. (2001) Computational Modelling of Flow around a Circular Cylinder in Sub-Critical Flow Regime with Various Turbulence Models. International Journal for Numerical Methods in Fluids, 35, 763-784. https://www.researchgate.net/publication/230271231_Computational_modelling_of _flow_around_a_circular_cylinder_in_sub-critical_flow_regime_with_various_turb ulence_models https://doi.org/10.1002/1097-0363(20010415)35:7<763::AID-FLD112>3.0.CO;2-S 
[24] Yakhot, V., Orszag, S.A., Thangam, S., Gatski, T.B. and Speziale, C.G. (1992) Development of Turbulence Models for Shear Flows by a Double Expansion Technique. Physics of Fluids A Fluid Dynamics, 4, 1510-1520.

https://doi.org/10.1063/1.858424

[25] Cheng, T.S. and Yang, W.J. (2008) Numerical Simulation of Three-Dimensional Turbulent Separated and Reattaching Flows using a Modified Turbulence Model. Computers \& Fluids, 37, 194-206.

http://www.sciencedirect.com/science/article/pii/S0045793007001168

[26] Wen, Z., Shi, L. and Ren, Y. (2009) Fluent Fluid Computing Application Tutorial. Tsinghua University Press, Beijing.

[27] Patankar, S.V. (1980) Numerical Heat Transfer and Fluid Flow. Hemisphere Pub. Corp.

[28] Pagalthivarthi, K.V., Gupta, P.K., Tyagi, V. and Ravi, M.R. (2011) CFD Prediction of Erosion in Centrifugal Slurry Pumps for Dilute Slurry Flows. Journal of Computational Multiphase Flows, 3, 225-246. https://doi.org/10.1260/1757-482X.3.4.225

[29] Roco, M.C. and Addie, G.R. (1983) Analytical Model and Experimental Studies on Slurry Flow and Erosion in Pump Casings. In: Proceedings of 8 th International Technical Conference on Slurry Transportation, Slurry Transport Association, Washington DC, 263. http://cat.inist.fr/?aModele=afficheN\&cpsidt $=9057590$

[30] Chen, B. and Peng, G. (1988) Study on the Clearance Flow of a Hydraulic Turbine Guide Vane. Journal of Lanzhou University of Technology, No. 1, 50-58. http://kns.cnki.net/KCMS/detail/detail.aspx?dbcode=CJFQ\&dbname=CJFD8589 